\title{
Preparation of 3D printed silver nanocomposites and their antibacterial properties
}

\author{
Chunli Zhu ${ }^{1, \dagger, *}$, Xiaofen $\mathrm{Fu}^{1, \dagger}$, Xing Gao ${ }^{1}$, Haotian Kan ${ }^{1}$ \\ ${ }^{1}$ School of Chemistry and Biological Engineering, Qilu Institute of Technology, Jinan, 250200, P. R. China. \\ ${ }^{\dagger}$ These two authors contribute equally to this work.
}

\begin{abstract}
The 3D printed antibacterial polymer products were rapidly sintered to obtain by selective laser sintering with highly dispersed Nano-silver modified Kaolin powder and polyamide/polypropylene composite engineering material. The preparation method of polymer products not only shortens the preparation period, but also guarantees the precision and quality of the product as well as the slow-release function of the Nanosilver.
\end{abstract}

\section{Introduction}

Bacteria, viruses and other microorganisms are all over every corner of human living environment. Bacteria proliferate rapidly, induce and infect diseases, which is an important factor threatening human health. Therefore, bacteriostatic and bactericidal materials have been the research focus in various fields [1-3]. Polymer material is a kind of material which is closely related to people's life. We have daily contact with all kinds of fabrics, containers, packaging materials and other daily necessities made of the polymer material, conventional polymer products do not have bacteriostatic properties, so they are easy to become a hotbed of bacteria. In order to produce antibacterial polymer products, some achievements have been made in recent years by adding various antibacterial agents into polymer materials and processing related products, among which the antibacterial materials containing Nano-silver have been studied widely [4-5],

This is because of the Nano-silver material prominent and stable antibacterial effect, and its large quantity of product. However, there is a difficult problem in the preparation of polymeric bacteriostatic materials containing Nano-silver, that is, the dispersion problem of Nano-silver, because Nano-silver is easy to agglomerate and cannot combine with polymer matrix uniformly. For this reason, people try to disperse Nano-silver first and then combine it with polymer materials [6].

There are many kinds of dispersion carriers for dispersing Nano-silver. Some colleges and research institutes have studied silicate carriers [7-8]. However there is no recognized high-efficiency and long-lasting silver-loaded silicate product available in the market at present, and there are still many obstacles in the industrialization manufacture of polymer bacteriostatic products. Therefore, a kind of 3D printed Nano-silver composite was prepared. The preparation process is simple and the material has excellent antibacterial property.

\section{Experiment}

\subsection{Materials and Equipment}

Polyamide, polypropylene, dimethyl sulfide, $\mathrm{AgNO}_{3}$, Polyvinylpyrrolidone, Kaolin powder, methanol, ethanol, Trisodium citrate, escherichia coli, UV lamp, drying box, mixer, vertical high speed mixer, 3D printer, analytical balance, photometer.

\subsection{Experiment section}

\subsubsection{Preparation of polymer materials}

The composite polymer material (CP) was prepared by mixing and pelleting polyamide and polypropylene according to the weight ratio of $1: 1$.

\subsubsection{Silver-loaded modified Kaolin}

The kaolin powder modified by dimethyl sulfoxide with a intercalation rate of more than $90 \%$ was added to the solution of $0.2 \mathrm{mmol} \mathrm{AgNO}_{3}$ and Polyvinylpyrrolidone. Then the mixed solution was uniformly stirred and then left for $3 \mathrm{~h}$ to prepare the precursor solution. The particle size range of Kaolin powder is $15 \mu \mathrm{m}$, the solution is ethanol solution with concentration not more than $15 \%$, the concentration of Polyvinylpyrrolidone in the solution is $0.5 \%$, and the mass ratio of Kaolin powder to solution is $1: 100$. Silver-loaded modified kaolin $(\mathrm{Ag}-\mathrm{Si})$ was prepared by adding $0.1 \mathrm{~mol} / 1$ trisodium citrate solution into the precursor solution, stirring it evenly, exposing it to UV lamp for $1.0 \mathrm{~h}$, then filtering it and drying it in a

*Corresponding author: Chunli Zhu; e-mail: chunli0125@163.com. 
drying oven at $80^{\circ} \mathrm{C}$ or less.

\subsubsection{D printing powder}

The composite 3D printing powder was prepared by mixing the composite polymer and silver-loaded modified Kaolin in a ball mill or a mixer with the weight ratio of 1:0.1.

\subsubsection{Preparation of macromolecule bacteriostatic material}

The substrate layer is printed with the prepared composite polymer material, and then the working layer is printed with the prepared composite $3 \mathrm{D}$ printing powder. The macromolecule antibacterial material (Ag-Si-CP) was obtained by layer-by-layer printing with selective laser sintering (SLS) according to the pre-set slicing procedure.

\subsection{Characterization}

\subsubsection{Morphological analysis}

The Crystal diffraction experiments were performed by Riau D/max-2500 X-ray diffractometer with a scanning speed of $10^{\circ} / \mathrm{Min}$ and a scanning range of $10^{\circ}-100^{\circ}$.

\subsubsection{Tensile test}

The tensile properties of the samples were tested by UTM5504X universal testing machine. Prior to testing, the sample material was printed by a 3D printer (Makerbot) into a preset dumbbell-shaped spline (effective length: $50 \mathrm{~mm} \times 10 \mathrm{~mm} \times 4 \mathrm{~mm}$ ).

\subsubsection{Thermogravimetric analysis}

The samples ( $\mathrm{CP}$ and $\mathrm{Ag}$-Si-CP) were weighed by an electronic balance and placed in a small crucible. The weightlessness of the samples was recorded by thermogravimetric analyzer (209 F3 Tarsus) at $\mathrm{N}_{2}$. The temperature range was $40{ }^{\circ} \mathrm{C}$ to $800{ }^{\circ} \mathrm{C}$, and the heating rate was $20^{\circ} \mathrm{C} / \mathrm{min}$.

\subsubsection{Antibacterial test}

The bacteriostatic experiment of the material was carried out by using Gram-negative (E.coli) in this study. E.coli was incubated at $37{ }^{\circ} \mathrm{C}$ for $24 \mathrm{~h}$. The OD600 of the suspension was determined by photometer. The concentration of the suspension was adjusted to (5.0-10.0) $\mathrm{x} 10^{5} \mathrm{cfu} / \mathrm{ml}$. In this experiment, the antibacterial property of the material was observed by sticking film method. The operation experiments were carried out according to the requirements of QB/T2591-2003. The steps are as follows: (1) the sample was cut into $20 \mathrm{~mm} \times 20 \mathrm{~mm}$ square slices. The surface of the sample was wiped with $70 \%$ alcohol solution, rinsed with sterile water for $1 \mathrm{~min}$, and then dried naturally. (2) The 30 ul test solution was added to the blank control sample and the antibacterial plastic sample respectively. The samples were covered with the film of sterilizing tweezers, and the bacteria were placed in the sterilizing plate and incubated the constant temperature incubator for $24 \mathrm{~h}$ at $37{ }^{\circ} \mathrm{C}$ and $\mathrm{RH}>90 \%$. (3) Saline of 3 $\mathrm{mL}$ containing $0.80 \% \mathrm{NaCl}$ was added to the cultured samples for $24 \mathrm{~h}$, and then the samples were rinsed repeatedly. After full shaking, a certain amount of liquid was inoculated on the solid medium with the inoculation ring, and cultured at $37^{\circ} \mathrm{C}$ for $24 \mathrm{~h}$, the number of colonies on the surface of solid medium was counted. (4) Cultured for $48 \mathrm{~h}$ under the same condition.

\section{Results and discussion}

\subsection{Morphology analysis of silver-loaded modified Kaolin}

Fig. 1 is the TEM images of silver-loaded modified Kaolin. From fig. 1, we can see that the scale of silver in the silverloaded kaolinite materials is Nano-scale, the particle size is $0.8 \mathrm{~nm}$, and the maximum is not more than $2.0 \mathrm{~nm}$. The parallel lattices of the nanoparticles were observed by HRTEM with a plane distance of about $2.4 \AA$ 。 The dimethyl sulfoxide modified Kaolin has been proved to be a highly dispersed carrier material for Nano silver particles, but at present, the bactericidal effect of silver nanoparticles loaded with dimethyl sulfoxide kaolin is not very good, because the bactericidal properties of silver nanoparticles are related to their size and release ability, and in the reduction process some strong reductant intervention also easy to limit its use. The control of particle size and yield of silver nanoparticles, especially the utilization of disodium citrate and light-assisted reduction, can be achieved by reasonable selection of reactant concentration and control of reduction conditions, the method not only shortens the reaction time but also avoids the excessive crystallization and growth of the Nano-silver, which ensures the output efficiency and the uniformity of the particle size.

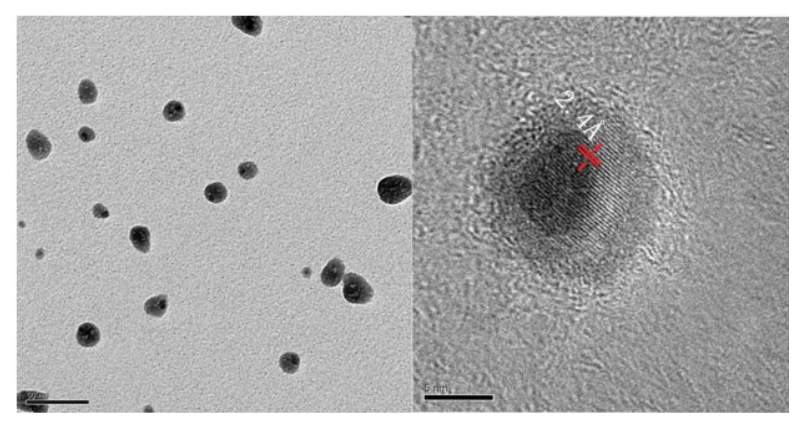

Fig 1. TEM images of silver-loaded modified Kaolin

\subsection{Tensile properties of macromolecular bacteriostatic material}

In the preparation of composite polymer materials, the combination of polyamide and polypropylene with a weight ratio of $1: 1$ can obtain better mechanical properties than each other, and can be well compatible in subsequent 3D printing. The mixing proportion of silver-loaded 
modified kaolinite can be controlled flexibly according to the practical needs, but it should be controlled so as not to affect the melting deposition of the Matrix material in 3D printing. The tensile strengths of $\mathrm{CP}$ and $\mathrm{Ag}-\mathrm{Si}-\mathrm{CP}$ composite materials were showed in Fig. 2. There is no significant difference in tensile properties between $\mathrm{CP}$ and $\mathrm{Ag}-\mathrm{Si}-\mathrm{CP}$. The reason is mainly due to the small amount of silver-loaded Modified Kaolin. At the addition of $10 \%$, the effect of Nano-silver on the mechanical properties of the composites is not significant.

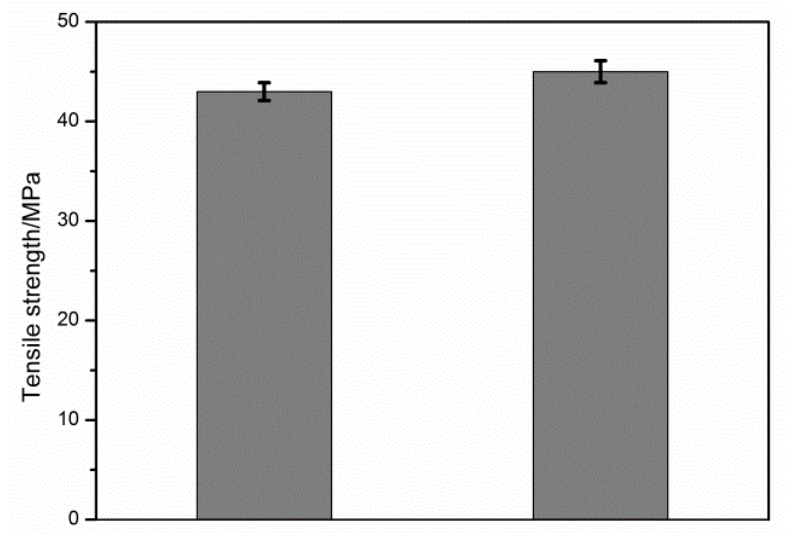

Fig 2. Tensile strengths of $\mathrm{CP}$ and $\mathrm{Ag}-\mathrm{Si}-\mathrm{CP}$ composite materials

\subsection{Thermal properties of Ag-Si-CP}

In order to meet the basic requirements of biomedical devices, the basic conditions include the ability to meet the use of high-temperature sterilization needs. Although the decomposition temperature of the composite is about $300{ }^{\circ} \mathrm{C}$, the possibility of introducing new materials into the system to change its thermal stability cannot be excluded. Therefore, the thermal properties are characterized and TGA curves are shown in Fig. 3. Compared with $\mathrm{CP}$, the composite material with Agmodified Kaolin did not decrease its TGA temperature, and the effect of Ag-modified Kaolin on the thermal properties of the composite material was not significant.

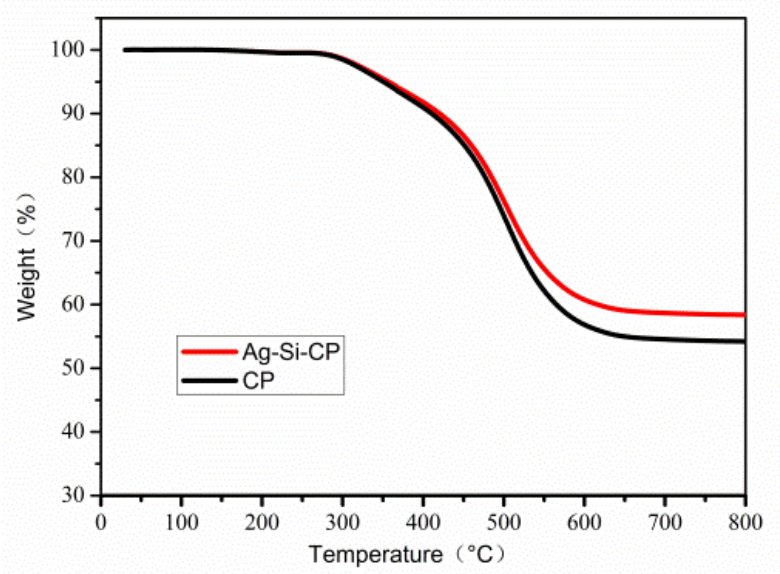

Fig 3. TGA of $\mathrm{CP}$ and $\mathrm{Ag}-\mathrm{Si}-\mathrm{CP}$ composite materials

\subsection{Antibacterial evaluation of Ag-Si-CP}

Fig. 4 shows the antibacterial properties of $\mathrm{CP}$ and Ag-Si$\mathrm{CP}$ composites against $\mathrm{E}$. coli. The white spots can be seen as colonies of E. coli from the eluent after $24 \mathrm{~h}$ of reculture. The control group is composite polymer material. It can be seen from the diagram that $\mathrm{CP}$ has no antibacterial property, but the addition of $10 \%$ silverloaded kaolinite material can obviously improve the antibacterial property of the material. When silver-loaded kaolinite was added, only a few colonies could be observed in $24 \mathrm{~h}$ and $48 \mathrm{~h}$, and the bacteriostatic rate was above $98.5 \%$, which indicated that the material had a good sustained-release effect.

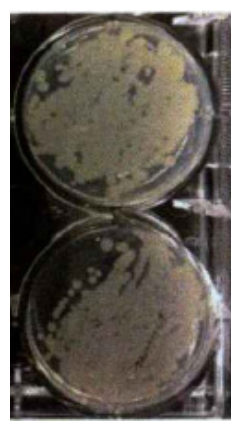

(a) CP $24 \mathrm{~h} \mathrm{CP} 48 \mathrm{~h}$

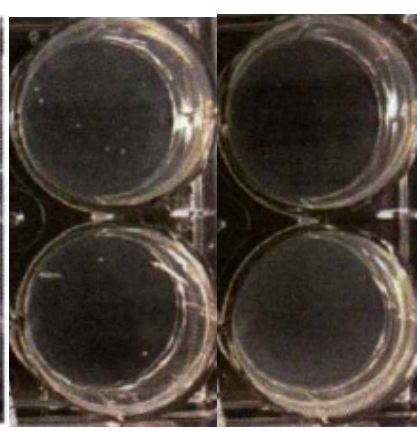

(b) Ag-Si-CP $24 \mathrm{~h}$ (c) Ag-Si-CP $48 \mathrm{~h}$

Fig 4. Antibacterial properties of $\mathrm{CP}$ and $\mathrm{Ag}-\mathrm{Si}-\mathrm{CP}$ composite materials

\section{Conclusions}

The 3D printing powder was prepared by mixing the composite polymer material with silver-loaded modified Kaolin material, and the composite 3D printing powder was printed layer by layer by selective laser sintering process. The combination of polyamide and polypropylene not only ensures the mechanical properties of the product, but also ensures the laser processability. By reasonably selecting the reactant concentration and controlling the reduction conditions in the preparation of silver-loaded Kaolin, the control of the particle size and the yield of the nano silver can be well realized, the operation time of the current technology from more than ten to dozens of hours can be shortened to a few hours to ensure the output efficiency. The diameter of silver nanoparticles can be as low as $0.8 \sim 2.0 \mathrm{~nm}$ and the particle size can be uniform combined with the optimized laser processing parameters, the precision and quality of the products as well as the slow-release function of silver nanoparticles can be guaranteed at the same time.

\section{Acknowledgments}

The authors are thankful to Research Project Foundation of Qilu Institute of Technology (No.

QL20K057) for financial support of this work.

\section{References}

1. $\mathrm{Ng} \mathrm{C}$, Goh S G, Saeidi N, etal. Occurrence of vibrio 
species, betalactam resistant vibrio species, and indicator bacteria in ballast and portwaters of a tropical harbor[J]. Science of the Total Environment, 2017, 651-656.

2. Guo S Z, Qiu K, Meng F, etal. 3D printed stretchable tactile sensors [J]. Advanced Materials, 2017, 29(27): 1218-1224.

3. Prorokova N P, Vavilova S Y, Kuznetsov O Y, etal. Antimicrobial properties of polypropylene yarn modified by metal nanoparticles stabilized by polyethylene [J]. Nanotechnologies in Russia, 2015, 10: $732-740$.

4. Jinwei Zhang, Yao Wang, etal. Preparation of high concentration Nano-silver and evaluation of its antibacterial effect [J]. Functional Material. 2020, 51(12): 12107-12111.

5. Leishan Shao, Jingjing Li, etal. Application of graphene/Nano-silver antibacterial agent in polypropylene [J]. Materials Science and Engineering. 2020, 36(11): 134-138.

6. Peng J M, Lin J C, etal. Enhanced antimicrobial activities of silver-nanoparticle-decorated reduced graphene nanocomposites against oral pathogens [J]. Materials Science and Engineering C, 2017, 71: 1016.

7. Cao G Z, Lin H, Kannan P, etal. Enhanced antibacterial and food simulant activities of silver nanoparticles/polupropylene nanocomposite films [J]. Langmuir, 2018, 34: 14537-14545.

8. Li T.Development of antibacterial agent at home and abroad and its application in PP [J]. China plastics Industry, 2003, 31(10): 5-8. 